This is the peer reviewed version of the following article: Wang, X., Li, X., Chu, X., Cao, R., Qian, J., Cong, Y., Huang, K., Wang, J., Redshaw, C., Sarangi, R., Li, G., Feng, S., Manipulating Surface Termination of Perovskite Manganate for Oxygen Activation. Adv. Funct. Mater. 2021, 2006439., which has been published in final form at https://doi.org/10.1002/adfm.202006439. This article may be used for non-commercial purposes in accordance with Wiley Terms and Conditions for self-archiving.

\title{
Manipulating Surface Termination of Perovskite Manganate for Oxygen Activation
}

Xiyang Wang, Xinbo Li, Xuefeng Chu, Rui Cao, Jingyu Qian, Yingge Cong, Keke Huang, Jiaao Wang, Carl Redshaw, Ritimukta Sarangi, Guangshe Li, and Shouhua Feng*

Dr. X. Wang, X. Li, Dr. J. Qian, Dr. Y. Cong, Prof. K. Huang, Prof. G. Li, Prof. S. Feng State Key Laboratory of Inorganic Synthesis and Preparative Chemistry, International Joint Research Laboratory of Nano-Micro Architecture Chemistry, College of Chemistry, Jilin University, Changchun 130012, People's Republic of China

E-mail: shfeng@jlu.edu.cn

Dr. X. Chu

Jilin Provincial Key Laboratory of Architectural Electricity \& Comprehensive Energy Saving, School of Electrical and Electronic Information Engineering, Jilin Jianzhu University, Changchun 130118, People's Republic of China

Dr. R. Cao, Dr. R. Sarangi

Stanford Synchrotron Radiation Light source SLAC National Accelerator Laboratory Menlo Park, California 94025, United States

J. Wang

School of Material Science and Engineering, University of Jinan, Jinan 250022, People's Republic of China

Prof. C. Redshaw

Plastics Collaboratory, Department of Chemistry, University of Hull, HU6 7RX, U.K.

Keywords: (perovskite oxides, surface segregation, electron structure, surface active site, oxygen activation)

\section{Abstract}

For $\mathrm{ABO}_{3}$ perovskite oxides, one of the key issues limiting their utilization in heterogeneous catalysis is the dominant presence of catalytically inactive A-site cations at the surface. The engineering of B-site terminated perovskite oxides is considered to be an effective method to address this issue, especially when dealing with $\mathrm{Mn} / \mathrm{Co}$-based perovskite catalysts. However, to-date, such a strategy has not been fully successful and remains a major challenge in the field. Herein, we have successfully synthesized a Mn-terminated perovskite manganate 
(B-LSM) via a one-pot hydrothermal method, in which low-valence Mn ions partially occupy the A site to form the active surface Mn-excess phase. Experimental results and theoretical calculations reveal that the presence of the Mn termination at the surface of the perovskite B-LSM optimizes the hybrid orbitals between Mn 3d and O 2p and promotes the activation of surface lattice oxygen, where the pristine inert lattice $\mathrm{O}^{2-}$ is evolved into active and stable lattice $\mathrm{O}^{2-x}$. Such structural optimization significantly reduces the activation energy barriers on going from $\mathrm{O}_{2}^{-}$species to important intermediate $\mathrm{O}^{-}$species during $\mathrm{O}_{2}$ activation. Moreover, this results in good stability and Pt-like activity for the Mn-terminated perovskite manganese oxide during $\mathrm{CO}$ oxidation. This work offers a new chemical route for the design of advanced perovskite-type oxides possessing novel functions.

\section{Introduction}

Perovskite-type materials possessing an $\mathrm{ABX}_{3}$ structure exhibit a wide variety of physical and chemical properties, such as photoelectricity, ferroelectricity, magnetoresistance, superconductivity and have been employed in heterogeneous catalysis. ${ }^{[1-4]}$ Furthermore, in recent years perovskite oxides have exhibited promise as catalysts in solid oxide fuel cells, vehicle emissions treatment, metal-air batteries, oxygen evolution and reduction reactions. This interest stems from their low cost, good thermal durability, unique electronic state, and broad tolerance to composition and structure. ${ }^{[5,6]}$ However, perovskite oxides have two inherent deficiencies, namely a small specific surface area and an inert surface segregation when compared to noble metal and simple oxide catalysts. This inevitably reduces both the number and the catalytic potential of the surface active sites and results in a severe underestimate of the true catalytic properties. ${ }^{[7]}$ Therefore, exploring suitable methods that can increase and activate the surface sites of perovskite oxides will play a significant role in the development and practical applications of perovskite-type catalysts going forward, mostly notably in the area of energy and the environment. 
Over the decades, many regulatory strategies have been developed to address this key problem, which involved increasing the spatial surface area, the introduction of appropriate defects (A-site or oxygen defects), the doping of different elements at the A and B sites, exposing high index crystal faces and tuning the crystal phase structure. ${ }^{[8-14]}$ Although the activity of these 'optimized' perovskite catalysts was improved to some extent, there still existed a fatal problem in that the outer surface of the regulated perovskites remained dominated by superabundant inactive A-site cations. ${ }^{[15]}$ It should be noted that although one monolayer AO segregation is beneficial for oxygen exchange kinetics, excess inert surface segregation seriously affects the activity and stability of perovskite oxides in catalytic reactions. In fact, such excesses are the key reason why it has proved so difficult to advance the catalytic performance of perovskite oxides. Recently, the passivation phenomenon has been confirmed by the use of a number of state-of-the-art techniques such as LEIS, AP-XPS, SRPES, AFM, and HAADF-STEM. The surface engineering of perovskite oxides is now an intense and challenging area of research. ${ }^{[16,17]}$

Neagu et al. pioneered the concept of perovskite exsolution, namely that B-site cations migrate from the bulk structure to the surface under a reducing atmosphere and finally precipitate into metal or alloy nanoparticles. ${ }^{[18]}$ This method not only increases the number of B-site active sites on the surface but also contributes to a stronger metal-oxide interface. Based on this technology, numerous new and advanced perovskite-type catalysts (e.g. $\mathrm{La}_{0.6} \mathrm{Sr}_{0.4} \mathrm{FeO}_{3-\delta},{ }^{[19]} \quad \mathrm{Sr}_{2} \mathrm{FeMo}_{0.65} \mathrm{Ni}_{0.35} \mathrm{O}_{6-\delta},{ }^{[20]} \quad \mathrm{La}_{0.43} \mathrm{Ca}_{0.37} \mathrm{Ni}_{0.06} \mathrm{Ti}_{0.94} \mathrm{O}_{3-\delta}{ }^{[21]}, \quad$ and $\operatorname{PrBaMn}_{1.8} \mathrm{Co}_{0.2} \mathrm{O}_{6-\delta}^{[22]}$ ) have been successfully prepared, where the catalytic performance and chemical durability has been greatly improved. However, these particular perovskites can only be applied in a reducing environment and the use of high-temperature annealing also leads to decreased spacial surface area. Notably, a series of surface modification techniques have been developed to overcome this limitation, and in particular it has been observed that decorating $\mathrm{Co}_{3} \mathrm{O}_{4}, \mathrm{HfO}_{2}, \mathrm{ZrO}_{2}$, and other perovskite oxides at the surface of a $\mathrm{La}_{1-x} \mathrm{Sr}_{x} \mathrm{CoO}_{3-\delta}$ 
thin film by pulsed laser deposition can effectively suppress phase separation thereby creating more active sites. ${ }^{[23,24]}$ Furthermore, some studies have also observed that surface segregation $\left(\mathrm{AO}_{x}\right)$ in perovskite oxides can be partly etched via the use of dilute acid, however the segregation will enrich again on the surface following prolonged reaction. ${ }^{[25,26]}$ Therefore, taking into account these observations, directly engineering B-site terminated perovskite oxides seems a much better way to enhance catalytic activity and therefore widen the potential practical applications of such systems. To date, a number of $\mathrm{TiO}_{2}$-terminated and $\mathrm{ZrO}_{2}$-terminated perovskite oxides have been prepared via pulsed laser deposition, molecular beam epitaxy, etc.., ${ }^{[27,28]}$ yet highly active $\mathrm{Mn} / \mathrm{Co}$-terminated perovskite oxides, which are viewed as some of the most promising alternative catalysts, are yet to be reported. Inspired by the pioneerig work that the $\mathrm{Mn}^{2+}$ atom with its large ionic radius can occupy the A-site of a perovskite structure under extreme conditions, ${ }^{[29-31]}$ an important breakthough to address surface A-ste segregation will be to target Mn occupied A-sites at the outer surface of perovskites.

In this paper, we report a one-pot synthetic method for accessing highly active Mn-terminated perovskite manganates for the first time and reveal that both non-stoichiometry and specific hydrothermal conditions are the key factors responsible for tuning the surface termination of perovskite oxides. The use of high-angle annular dark-field scanning transmission electron microscopy, X-ray absorption, X-ray photoelectron, and synchrotron radiation photoelectron spectroscopy reveal that a low-valent $\mathrm{Mn}$ atom rather than the traditional $\mathrm{La} / \mathrm{Sr}$ atom occupies the $\mathrm{A}$ site at the perovskite outer surface, and this ultimately results in active $\mathrm{MnO}_{x}$ segregation. On comparison with the perovskite $\mathrm{La}_{0.5} \mathrm{Sr}_{0.5} \mathrm{MnO}_{3}$ with A-site segregations (A-LSM), the Mn-terminated perovskite $\mathrm{La}_{0.45} \mathrm{Sr}_{0.45} \mathrm{MnO}_{3}$ (B-LSM) exhibits higher activity and stability, and this facilitates the complete conversion of carbon monoxide to carbon dioxide at $215^{\circ} \mathrm{C}$. Furthermore, the $\mathrm{O}$ K-edge XANES, $\mathrm{O}$ 1s XPS, $\mathrm{H}_{2}$-TPR, and $\mathrm{O}_{2}$-TPD show that the Mn-excess phase 
prominently improves the activity of the surface oxygen sites, i.e. pristine inert lattice oxygen $\mathrm{O}^{2-}$ is transformed into active and stable lattice oxygen $\mathrm{O}^{2-x}$. Theoretical calculations confirmed that these abundant self-regenerating surface active oxygen sites in B-LSM can optimize the activation pathway in the $\mathrm{CO}$ oxidation reaction and reduce the activation energy barrier of the oxygen species, which is the main reason for the observed improvement in the catalytic activity and stability.

\section{Results and discussion}

Segregation and/or phase separation in perovskite oxides is a common phenomenon, and is crucial to their performance in environmental catalysis and energy conversion. ${ }^{24}$ However, perovskite samples prepared by either the traditional solid-phase or sol-gel methods are usually formed in their thermodynamically stable phase, whilst the highly active B-site terminated perovskite oxides are not accessible. In contrast to the diffusion type mechanism in these methods, the hydrothermal synthetic approach allows for various growth mechanisms and can thus be used to prepare intermediate, metastable, and spacial phases. ${ }^{[32]}$ Studies on the hydrothermal synthesis of perovskite oxides has shown that the crystal growth is via a dehydroxylation process in a strong alkaline solution. In our previous work, we successfully prepared perovskite manganate with different crystal facets and further demonstrated that by introducing appropriate tetrahedral configuration in the form of cationic $\mathrm{NH}^{4+}$, the latter can form the linkage $\mathrm{N}-\mathrm{H} \cdots \mathrm{O}-\mathrm{B}$ enabling the modulation of high-energy crystal facets. ${ }^{[33]}$ Furthermore, it has also been reported that A-sites in perovskite oxides can be occupied by $\mathrm{Mn}^{2+}$ atom of large ion radius under high-pressure conditions. Inspired by such results, we investigated the use of hydrothermal experiments to achieve the targeted synthesis of Mn-terminated perovskite oxides. As shown in Figure 1a, the A-site terminated perovskite $\mathrm{La}_{0.5} \mathrm{Sr}_{0.5} \mathrm{MnO}_{3}$ was successfully prepared for the first time using the traditional hydrothermal method (Experimental details are shown in the Supporting Information). Sufficient La and Sr atoms in an alkaline solution can facilitate the crystal growth of the perovskite $\mathrm{La}_{0.5} \mathrm{Sr}_{0.5} \mathrm{MnO}_{3}$ 
(A-LSM) and are finally enriched on the surface to develop a stable structure $\left(\mathrm{AO}_{x}\right)$. Thus, the surface morphology of the perovskite $\mathrm{La}_{0.5} \mathrm{Sr}_{0.5} \mathrm{MnO}_{3}$ (A-LSM) prepared by this method is generally the bulk material present (Figure S1a SEM and Figure S1c TEM). To synthesize Mn-terminated perovskite manganate, we reduced by about $10 \%$ the raw materials for the elements $\mathrm{La}$ and $\mathrm{Sr}$ according to our design method, namely, the chemical formula of $\mathrm{La}_{0.45} \mathrm{Sr}_{0.45} \mathrm{MnO}_{3}$ (Figure 1b). The low concentration of $\mathrm{La}$ and $\mathrm{Sr}$ ions in perovskite $\mathrm{La}_{0.45} \mathrm{Sr}_{0.45} \mathrm{MnO}_{3}$ (B-LSM) has an important effect on its growing process. When the La and $\mathrm{Sr}$ atoms in solution are completely consumed, the remaining $\mathrm{Mn}$ atom will continue to grow at the surface. Given that the formation of $\mathrm{KMnO}_{3}\left(\mathrm{Mn}^{5+}\right.$ for $\mathrm{B}$ site and $\mathrm{K}^{+}$ions for A site) on the surface requires higher energy, the low-valent $\mathrm{Mn}$ atom is more likely to occupy an A-site and form several atomic layers of $\mathrm{MnO}_{x}$ segregation at the outer surface under high-pressure conditions. The result is not only decreased surface free energy but also the prevention of further growth of perovskite oxide crystals. Moreover, some studies have demonstrated that manganese oxides $\mathrm{Mn}_{2} \mathrm{O}_{3}$ can form a perovskite structure and that the $\mathrm{Mn}$ atom occupies the A site in the perovskite structure, which confirms our experimental hypothesis. ${ }^{[29,31,34]}$ Based on these design principles, our Mn-terminated perovskite $\mathrm{La}_{0.45} \mathrm{Sr}_{0.45} \mathrm{MnO}_{3}$ (B-LSM) will possess small crystal size and a unique $\mathrm{MnO}_{x}$-terminated surface structure, different from the A-LSM prepared via the conventional hydrothermal method.

To demonstrate the proposed structure, we measured the surface morphology of both A-LSM and B-LSM using SEM and TEM. As displayed in Figures S1, S2 and S3, B-LSM exhibits smaller crystal size ( $\sim 100 \mathrm{~nm}$ nanoparticle) versus A-LSM ( $\sim \mu$ m polycrystal). After grinding using an agate mortar, the crystal size of A-LSM is reduced to $\sim 1.5 \mu \mathrm{m}$. Furthermore, the crystal phase of A-LSM and B-LSM was analyzed by X-ray diffraction. Figure 2a shows that A-LSM and B-LSM have the same crystal structure each possessing the space group Pm3m. All diffraction peaks indexed to (100), (110), (111), (200), (210), (211), 
(220), (300), (310), and (311) for cubic perovskite $\mathrm{La}_{0.5} \mathrm{Sr}_{0.5} \mathrm{MnO}_{3}$, respectively. However, there are two significant differences in the XRD spectra between A-LSM and B-LSM. Firstly, the peak intensity for B-LSM is weaker than that of A-LSM; secondly, the diffraction peaks of B-LSM shift to low angle compared to A-LSM. These results indicate that B-LSM has lower crystallinity and higher structural disorder, and that B-LSM also exhibits proper lattice expansion. To confirm these results, we also measure the HRTEM images for both A-LSM and B-LSM by transmission electron microscopy (TEM). Figures S1e and f reveal that they are pure perovskite phase structures, with B-LSM having the larger lattice spacing $(2.76 \AA)$ versus A-LSM (2.74 $\AA$ ) in the (110) crystal facet (calculated in Figures S4 and S5). These results are consistent with the experimental XRD results above.

To visualize directly the surface atomic structure of the perovskite samples, high-angle annular dark-field scanning transmission electron microscope (HAADF-STEM) was performed. It is well known that the intrinsic surface of perovskite oxides is usually dominated by A-site cations, ${ }^{[15,16]}$ and the HAADF-STEM measurements in Figure S6 also revealed that surface termination is dominated by A-site cations of large ionic radius. In the case of the B-LSM sample (Figure 2b), it was evident that the outer surface of B-LSM was predominantly enriched with few layers of $\mathrm{Mn}$ atoms. Figure 2c presents a magnified simulation image at the surface of B-LSM, where surprisingly it is observed that an Mn atom occupies the A site in the perovskite structure whilst at the outer surface exists $\mathrm{MnO}_{x}$ segregation of 1 2 atomic layers. Figure 2d also confirms that surface termination of perovskite B-LSM involves an unusual $\mathrm{Mn}$ segregation. To further confirm these observations, we also performed surface atom arrangement of different samples and positions (Figure S7). These experimental results confirmed the presence of excess $\mathrm{Mn}$ atoms at the outer surface of the perovskite oxides and the crystal structure of B-LSM.

Synchrotron radiation photoelectron spectroscopy (SRPES) is another important detection tool for analyzing the outer surface structure of solid materials and the shallowest depth it can 
detect is $\sim 0.4 \mathrm{~nm}(\sim 4 \AA, 2-4$ atomic layers $) .{ }^{[35,36]}$ The surface composition and structure of A-LSM and B-LSM at different depths were investigated by photon energy (hv), which allows for changes in composition to be observed at the atomic level. Considering the binding energy of these elements, we measured spectra such as La 4d, Sr 3d, and Mn 3p and the experimental photon energies were $190 \mathrm{eV}, 280 \mathrm{eV}, 400 \mathrm{eV}$ and $1253 \mathrm{eV}$, respectively. The mean free paths (MFPs) for the photoelectron excitations for these materials at these three energies are approximately $0.4,0.7,0.9$, and $1.6 \mathrm{~nm}$, respectively. ${ }^{[35]}$ Furthermore, the XPS data in Figures S8-S13 are normalized and integrated in order to analyze their surface composition content. Although the ionization cross sections and binding energy for La, Sr, and $\mathrm{Mn}$ are different, their content can be also qualitatively analyzed. As shown in Figure 3a, the surface $(\mathrm{La}+\mathrm{Sr}) / \mathrm{Mn}$ fraction of B-LSM is lower than that of A-LSM at different depths and the $(\mathrm{La}+\mathrm{Sr}) / \mathrm{Mn}$ fraction also has a drastic improvement on increasing the probe depth in perovskite samples, resulting in double peaks for the elements La and Sr. In the outer surface (2 6 atomic layers) of both A-LSM and B-LSM at a kinetic energy of $190 \mathrm{eV}$, we observe that the $(\mathrm{La}+\mathrm{Sr}) / \mathrm{Mn}$ fraction for B-LSM is less than $1(\sim 0.9)$, while that for A-LSM is $\sim 1.9$. When the photo energy of the measurement reaches $280 \mathrm{eV}$, it eliminates surface interference for multi-angle scattering power samples and surface adsorption species. Therefore, the $(\mathrm{La}+\mathrm{Sr}) / \mathrm{Mn}$ fraction of A-LSM is about four times bigger than that of B-LSM at $280 \mathrm{eV}$. These results indicate that more Mn atoms in B-LSM occur at the outer surface. In addition, we can also estimate the amount of A-site and B-site elements in A-LSM and B-LSM and thereby confirm the surface composition and structure. As displayed in Figure $\mathbf{3 b}$, as the depth increases, the A-site content at the surface for A-LSM and B-LSM first increases and then flattens out, and the surface composition of B-LSM gets closer and closer to that of A-LSM. These results suggest that B-LSM possesses more Mn atoms at the outer surface. Based on these results, we speculate that the A-site at the outer surface of B-LSM may be occupied by a low-valent-state Mn atom. Considering this, Mn 3p XPS for A-LSM and 
B-LSM were investigated at different surface depths. As shown in Figure 3c, the Mn species at the outer surface of A-LSM is assigned to perovskite termination with higher binding energy $(51.5 \mathrm{eV})$. On increasing the probe depth, the valence state of the Mn atom first decreases $(51.3 \mathrm{eV})$ and then does not changed much due to an increase of bulk structure, indicating that A-LSM only contains perovskite-related Mn species. In contrast, the Mn atom at the outer surface of B-LSM (Figure 3d) exhibits a lower valence state $(50.4 \mathrm{eV})$, resulting in $\mathrm{MnO}_{x}$ segregation and an A-site $\mathrm{Mn}$ atom in a lower oxidation state. On increasing the probe depth, the emergence of perovskite termination and the bulk structure improves the average valence state of $\mathrm{Mn}$ atom and its $\mathrm{Mn} 3 \mathrm{p}$ peak position remains unchanged, demonstrating that for B-LSM there exists various Mn species possessing different chemical environments. The occurrence of broad half-peak and high peak intensity on lower binding energy for B-LSM at the photo energy for $1253 \mathrm{eV}$ confirms that B-LSM contains more low-valence-state Mn species compared to A-LSM. These results are also consistent with the HAADF-STEM data and further clarify that Mn-terminated LSM is successfully prepared by our synthetic methodology.

To further verify the surface electronic structure and composition of these samples, X-ray photoelectron (XPS) and soft X-ray absorption near-edge structure (XANES) spectroscopic experiments were performed. Figure S14 and Figures 4a and b present La 3d, Sr 3d, Mn 2p, Mn 3s, and O 1s XPS data for A-LSM and B-LSM. It can be observed that La 3d and Sr 3d XPS for B-LSM possess smaller peak intensity and integral area than does A-LSM (Figure S14a and b), whilst Mn 2p XPS for B-LSM in Figure S14c exhibits the larger peak intensity and integral area, confirming that B-LSM is different from the traditional A-site cationic enriched perovskite oxides (A-LSM) and contains more B-site elements at the surface. Moreover, the composition content of these samples has been estimated by XPS, ICP, and EDS quantitative analysis. As shown in Table 1, the XPS results reveal that A-LSM contains $32.9 \% \mathrm{La}, 30.9 \% \mathrm{Sr}, 36.2 \% \mathrm{Mn}$ at the surface, whilst the results for B-LSM are 
$21.9 \%, 22.5 \%$, and $55.6 \%$, respectively. B-LSM has a lower ratio $(0.95)$ of $(\mathrm{La}+\mathrm{Sr}) / \mathrm{Mn}$ than does A-LSM (1.76), which intuitively indicates that there are more B-site active sites for B-LSM at the surface. Inductive Coupled Plasma Emission Spectrometer (ICP) is used to measure the total element content of a sample. Herein, ICP results reveal that B-LSM possesses a lower ratio (0.93) for $(\mathrm{La}+\mathrm{Sr}) / \mathrm{Mn}$ versus A-LSM (1.08), agreeing well with our experimental dosage for the $\mathrm{La}, \mathrm{Sr}$, and Mn metal salts. Meanwhile, the $\mathrm{Sr} /(\mathrm{La}+\mathrm{Sr})$ ratio for both A-LSM and B-LSM was calculated using the ICP data and as shown in Table 1 they are 0.49 (A-LSM) and 0.52 (B-LSM), respectively. This result is also consistent with the theoretical value for the chemical stoichiometric ratio. In addition, Energy Dispersive Spectrometer (EDS) (Figures S15 and S16) provides similar experimental results, namely that the ratio of $(\mathrm{La}+\mathrm{Sr}) / \mathrm{Mn}$ for A-LSM is 1.46 and that for B-LSM is 1.24.

In the previous sections, we have demonstrated that the surface termination of the prepared B-LSM is that of B-site elements rather than the traditional A-site element, and that its surface also has more Mn active sites. A more in-depth study of the surface electronic structures of A-LSM and B-LSM was then undertaken. Figure S14c shows that the Mn 2p peak of B-LSM shifts to a lower bind energy compared to A-LSM, indicating a decrease in the oxidation state for the Mn atom. The Mn 3s XPS is also used to half-quantitatively analyze the valence state. In Figure S14d, the energy separations of A-LSM and B-LSM are 4.5 and $5.2 \mathrm{eV}$, respectively. According to the literature, ${ }^{[37,38]}$ the average valence state for the Mn element for A-LSM and B-LSM is $\sim 3.8+$ (surface $\mathrm{La} / \mathrm{Sr}$ enrichment) and $\sim 3.2+$ (surface Mn enrichment), respectively. The decrease in the valence state can be attributed to the occupation of the A-site in the outer surface of B-LSM by an Mn atom with a lower oxidation state. Furthermore, the O 1s XPS of A-LSM and B-LSM were carried out to examine the surface oxygen species. As shown in Figures $4 \mathbf{a}$ and $\mathbf{b}$, the $\mathrm{O}$ 1s spectrum is divided into four components, namely bulk $\mathrm{O}^{2-}\left(\mathrm{O}_{\text {lattice }}\right)$ located at $\sim 528.7 \mathrm{eV}$, perovskite lattice termination at $\sim 529.5 \mathrm{eV}$, surface oxygen species $\left(\mathrm{O}_{\text {surface }}\right)$ at $\sim 531.2 \mathrm{eV}$ and surface adsorbed oxygen species $\left(\mathrm{O}_{\text {surface }}\right)$ at $\sim 533.6$ 
eV. ${ }^{[39,40]}$ Table 1 reveals that the surface oxygen/lattice oxygen ratio of B-LSM is 2.73, which is more than that of A-LSM (1.26), which indicates that the surface of B-LSM has absorbed more oxygen species, for example $\mathrm{H}_{2} \mathrm{O}$ and $\mathrm{CO}_{2}$. Moreover, as shown in Figures 4a and b, and Figure S17, the O 1s XPS peak of B-LSM for the perovskite lattice termination also shifts to high energy, indicating the presence of more active lattice oxygen $\left(\mathrm{O}^{2-x}\right)$ in B-LSM.$^{[41]}$ These results clearly illustrate that B-site termination can dramatically increase the activity of any surface oxygen species present and can promote the activation of molecular oxygen in oxidation reactions.

The O K-edge and Mn L-edge XAS were measured to study the spin state, covalent character of the bonding, and the electron density at the outer surface. ${ }^{[41]}$ In Figures $4 \mathbf{c}$ and d, the O K-edge and Mn L-edge XAS for A-LSM and B-LSM are normalized from 518 to 557 $\mathrm{eV}$ and from 630 to $662 \mathrm{eV}$, respectively. As shown in Figure 4c, peak a and peak b at the O $\mathrm{K}$-edge are assigned as the hybridization between $\mathrm{Mn} 3 \mathrm{~d}$ and $\mathrm{O} 2 \mathrm{p}$; peak $\mathrm{c}$ is considered as a surface oxygen species; peak d derives from the hybridization between $\mathrm{La} 5 \mathrm{~d} / \mathrm{Sr} 4 \mathrm{~d} / \mathrm{Mn} 4 \mathrm{sp}$ and $\mathrm{O} 2 \mathrm{p} \cdot{ }^{[41]}$ Compared with A-LSM, the peak a in B-LSM shifts to a high binding energy and its peak intensity becomes weaker, which is ascribed to the enhanced covalency of the Mn-O bond. Moreover, the intensity of peak $b$ increased significantly and the peak position also shifted to high binding energy, and this was attributed to higher covalence and increased $\mathrm{MnO}_{x}$ surface segregations. High energy shifts for peak c suggested a transition from $\mathrm{O}_{2}{ }^{-}$ species $\left(\mathrm{O} 1 \mathrm{~s} \rightarrow 3 \sigma_{\mathrm{u}}\right)$ to $\mathrm{O}_{2}^{-1+x}$ species $\left(\mathrm{O} 1 \mathrm{~s} \rightarrow 3 \sigma_{\mathrm{u}}\right)$, demonstrating that the surface lattice oxygen of B-LSM was successfully activated, ${ }^{[41]}$ which is in agreement with the O 1s XPS results. In contrast to the $\mathrm{O} \mathrm{K}$-edge XAS, the $\mathrm{Mn} \mathrm{L}_{2}, \mathrm{~L}_{3}$-edge XAS in Figure 4d reveals no positional change for the absorption peak, whilst the white line intensity of B-LSM becomes weaker compared to A-LSM, suggestive of either low electron density for the Mn atom or high covalency for the Mn-O bond. To confirm these inferences, we also measured the Mn K-edge XAS via XANES and EXAFS for A-LSM and B-LSM ${ }^{[42]}$ The normalized Mn K-edge 
XANES spectrum is shown in Figure 4e, and it is observed that the X-ray absorption edge of B-LSM shifts to a low energy compared to A-LSM, again suggestive of a lower average oxidation state for the Mn atom. For the electronic structure of Mn 3d (Mn 1s $\rightarrow$ Mn 3d), B-LSM also displayed a weak intensity for the Mn 3d absorption peak, consistent with the Mn L-edge XAS spectrum. These results further prove the existence of an $\mathrm{MnO}_{x}$ phase or an $\mathrm{Mn}$ atom at the A-site. To clarify the local structure of the Mn atom in the perovskite oxides, the Mn K-edge EXAFS spectrum of A-LSM and B-LSM was transformed by K-space and R-space Fourier-transformed FT $\left(\mathrm{k}^{3} \chi(\mathrm{k})\right)$. The K-space functions shown in Figure $\mathbf{S 1 8}$ reveal that there is a similar peak shape and high signal-to-noise ratio for samples of both A-LSM and B-LSM, implying both adopt the same crystal structure. However, there are some clear differences between these two samples in the R-space function, see Figure 4f. Firstly, the Mn-O bond length in B-LSM $(\sim 1.53 \AA)$ is longer that that observed for A-LSM $(\sim 1.49 \AA)$; secondly, the Mn-La/Sr/Mn bond distance in B-LSM is also longer than that in A-LSM, and the peak intensity has marked decreased. Thirdly, the marked blue area clarifies that B-LSM contains some unusually short Mn-Mn (A-site in LSM) bonds. These experimental results are consistent with evident B-LSM lattice expansion, a higher disorder degree in the local environment of $\mathrm{Mn}-\mathrm{La} / \mathrm{Sr} / \mathrm{Mn}$ bond, and a small amount of $\mathrm{Mn}-\mathrm{Mn}$ (B-site - A-site) bonding. Importantly, the XRD, TEM, and XAS results are consistent and are also mutually validated. In addition, the fitting curves ( $\mathrm{K}$ space and $\mathrm{R}$ space) and parameters $\left(\mathrm{CN}, \mathrm{R}(\AA), \Delta \mathrm{E}_{0}(\mathrm{eV}), \sigma^{2}\right.$ $\left(10^{-3} \AA^{2}\right)$, R factor) of EXAFS spectra for A-LSM and B-LSM was also carried out (Figures S19-S22 and Table S1). There is good overlap between these fitting effect curves and the experimental data, and the corresponding fitting parameters are also consistent with the above explanation.

$\mathrm{H}_{2}$-TPR was carried to analyze the chemical adsorption capacity and redox behavior of the solid catalysts. The $\mathrm{H}_{2}$-TPR profiles for A-LSM and B-LSM are illustrated in Figure 5a. In 
the case of the A-LSM sample, there are two main reduction peaks at $498.7^{\circ} \mathrm{C}$ and $710.6{ }^{\circ} \mathrm{C}$, corresponding to the reduction processes: 1$) \mathrm{Mn}^{4+} \rightarrow \mathrm{Mn}^{3+}\left(\sim 470{ }^{\circ} \mathrm{C}\right)$, and 2) $\mathrm{Mn}^{3+} \rightarrow \mathrm{Mn}^{2+}$ $\left(\sim 720{ }^{\circ} \mathrm{C}\right) .{ }^{6}$ While B-LSM shows two reduction peaks for the $\mathrm{Mn}^{4+}$ ions at low temperature and a higher reduction temperature for $\mathrm{Mn}^{3+} \rightarrow \mathrm{Mn}^{2+}\left(736.9{ }^{\circ} \mathrm{C}\right)$. The double peak phenomenon may stem from the presence of two kinds of $\mathrm{Mn}^{4+}$ species at the surface and in the bulk of the B-LSM and the low reduction temperature results in outstanding redox activity. Furthermore, B-LSM also exhibits a lower reduction onset peak temperature (239.3 $\left.{ }^{\circ} \mathrm{C}\right)$ compared to A-LSM $\left(265.2{ }^{\circ} \mathrm{C}\right)$, indicating that the surface lattice oxygen in B-LSM is more active. In addition, it was observed that the $\mathrm{H}_{2}$-TPR peak for A-LSM shifted to higher T compared with B-LSM, which can be attributed to the different particle size and surface site activity. Specifically, a longer diffusion length within the particles in A-LSM increases the reduction temperature of the sample and the poor surface site activity in A-LSM also improves the difficulty of $\mathrm{H}_{2}$ adsorption and reduction. The quantitative analysis results for $\mathrm{H}_{2}$ consumption were also calculated (Table 1), and it is evident that B-LSM has higher total $\mathrm{H}_{2}$ consumption (2.39 $\left.\mathrm{mmol} \mathrm{g}^{-1}\right)$ versus A-LSM (2.02 $\left.\mathrm{mmol} \mathrm{g}^{-1}\right)$, which derives from the different chemical formulas and surface structures. These results are in close agreement with the ICP, EDS, and XPS data. $\mathrm{O}_{2}$-TPD was also performed to study the oxygen species associated with these solid catalysts. The oxygen species are typically divided into adsorbed oxygen $\left(\mathrm{O}_{\mathrm{ads}}\right.$, $\left.<400{ }^{\circ} \mathrm{C}\right)$ and lattice oxygen $\left(\mathrm{O}_{\text {latt }},>400{ }^{\circ} \mathrm{C}\right) .{ }^{[3]}$ In Figure 5b, B-LSM has a lower desorption temperature $\left(81.8^{\circ} \mathrm{C}\right)$ for adsorbed oxygen and lattice oxygen $\left(452.3,593.4,798.5^{\circ} \mathrm{C}\right)$ versus A-LSM $\left(90.4,548.7,682.7^{\circ} \mathrm{C}\right)$. Meanwhile, the onset temperature for B-LSM is clearly lower than that of A-LSM and the desorption capacity (desorption peak area) for B-LSM is 
also higher, confirming that B-LSM possesses more active oxygen species and strong lattice oxygen mobility. This is also affirmed by the $\mathrm{O} 1 \mathrm{~s}$ XPS, O K-edge XAS, and $\mathrm{H}_{2}$-TPR.

To evaluate the effect of surface termination on catalytic activity, $\mathrm{CO}$ oxidation measurements for A-LSM and B-LSM were performed. As shown in Figure 5c, the CO oxidation activity of A-LSM is inferior, while B-LSM has Pt-like catalytic activity whereby carbon monoxide can be completely converted into carbon dioxide at $215^{\circ} \mathrm{C} .{ }^{43,44}$ Specifically, as shown in Table 1, B-LSM has lower conversion temperatures $T_{10}, T_{50}$, and $T_{90}(117,164$, and $\left.196{ }^{\circ} \mathrm{C}\right)$ compared to A-LSM $\left(218,283\right.$, and $\left.368^{\circ} \mathrm{C}\right)$. Furthermore, the specific surface area of A-LSM and B-LSM was measured via a nitrogen adsorption apparatus. The BET of A- LSM and B-LSM is $0.85 \mathrm{~m}^{2} \mathrm{~g}^{-1}$ and $2.65 \mathrm{~m}^{2} \mathrm{~g}^{-1}$, respectively (Table 1). To eliminate the effect of surface area, $\mathrm{CO}$ oxidation reaction rates normalized by catalyst surface area were calculated to enable a study of the intrinsic catalytic activity of A-LSM and B-LSM. Figure 5d shows that B-LSM has higher reaction rates $\left(\mathrm{mol} \mathrm{s}^{-1} \mathrm{~m}^{-2}\right)$ at different temperatures compared to A-LSM, indicating higher intrinsic catalytic activity for B-LSM. Furthermore, temperature-dependent turnover frequencies (TOFs) for $\mathrm{CO}$ oxidation were also calculated to evaluate the catalytic performances of both A-LSM and B-LSM. As shown in Figure 5e, B-LSM exhibits higher turnover frequencies (TOFs: $\mathrm{S}^{-1}$ ) for $\mathrm{CO}$ oxidation compared to A-LSM under the same reaction conditions; TOFs are normalized by the number of Mn active sites on the catalyst surface. TOFs normalized by the molar quantities of the samples are displayed in Figure S23. In addition, the activation energy of A-LSM and B-LSM for CO oxidation was calculated (see experimental section of supporting information). As shown in Figure S24, B-LSM possesses a lower activation energy of $\sim 29.43 \mathrm{~kJ} / \mathrm{mol}$ compared with A-LSM $(\sim 56.53 \mathrm{~kJ} / \mathrm{mol})$. These results unanimously prove that B-site metal cations are veritable reactive sites and B-LSM has a more intrinsic catalytic property than A-LSM. In addition, the stability of the systems for $\mathrm{CO}$ oxidation was measured with runs performed at $196{ }^{\circ} \mathrm{C}$ and $370{ }^{\circ} \mathrm{C}\left(\sim 90 \% \mathrm{CO}\right.$ conversion) for $48 \mathrm{~h}$ at a flow rate of $50 \mathrm{~mL} \mathrm{~min}{ }^{-1}$, 
respectively. From Figure 5f, it can be observed that the catalytic activity of perovskite A-LSM markedly decreases on increasing the reaction time, whilst B-LSM exhibits excellent stability and readily maintains $90 \%$ CO conversion after enduring $48 \mathrm{~h}$ measurements and only decreases $2.12 \%$ in its catalytic performance.

To further comprehend the effect of surface termination on activity and stability, XPS analysis of A-LSM and B-LSM after the catalytic reaction was performed to study the fundamental reason for the stability during the reaction process. As shown in Figures S25-S27, the C 1s XPS spectra of A-LSM after catalytic reaction (Figure S25a) exhibit an obvious peak for $\mathrm{CO}_{3}{ }^{2-}$ at $\sim 290 \mathrm{eV}$, whereas for B-LSM there is no sign of such a peak under similar catalytic conditions, which indicates that $\mathrm{CO}_{3}{ }^{2-}\left(\mathrm{SrCO}_{3}\right)$ can readily accumulate at the surface of A-LSM and its coverage can reduce surface active sites, while B-LSM has strong desorption ability for $\mathrm{CO}_{2}$ molecules due to the presence of more self-regenerating active sites on the surface (less surface segregation $\mathrm{AO}_{x}$ ). Furthermore, the $\mathrm{O} 1 \mathrm{~s}$ XPS data for these samples post-reaction was also performed in order to analyze the activity of the surface oxygen species. As displayed in Figure S25 b-d, A-LSM has more carbonate on the surface than does B-LSM (and the original A-LSM) following the catalytic reaction, while carbonate absorbed at the surface of B-LSM underwent no obvious change during the catalytic process, consistent with the C 1s XPS data (Figure S25a). Importantly, the lattice oxygen position in B-LSM is still higher than that of A-LSM after the catalytic reaction and primal B-LSM, indicating that the high-activity lattice oxygen in B-LSM has preeminent reversible cyclability and is also the key reason for the observed prolonged stability during the catalytic reaction. The Mn 2p, Mn 3s, La 3d, and Sr 3d XPS results (Figures S26 and S27) reveal that after the catalytic reaction, the oxidation state of the Mn atom in A-LSM remains unchanged at $\sim 3.8$, whilst that for B-LSM increased from $\sim 3.2$ to $\sim 3.38$, confirming the reconstruction of surface $\mathrm{MnO}_{x}$, and this is also in good agreement with the surface lattice oxygen activity in the $\mathrm{O} 1 \mathrm{~s}$ XPS. In addition, the data also demonstrate that B-LSM after reaction still contains a high 
content of the catalytic active Mn element compared with A-LSM. Poor durability for A-LSM derives from the enrichment of inactive A-site cations and carbonate $\left(\mathrm{CO}_{3}{ }^{2-}\right)$ at the surface during the $\mathrm{CO}$ catalytic oxidation process, covering surface reactive sites and thereby decreasing the activity at the surface. By contrast, B-LSM contains more self-regenerating active sites. Less carbonate and important Mn segregations in B-LSM can effectively activate the surface lattice oxygen without crippling the surface reactive sites, and this is the fundamental reason why B-LSM exhibits good activity and stability.

To better understand the key role played by surface termination on the catalytic process of CO oxidation, density functional theory (DFT) calculations were applied to describe the reaction pathway and define the rate-limiting step. The computational details for the $\mathrm{CO}$ oxidation are given in the experimental section in supporting information and Figures S28 and S29. As shown in Figure 6, according to the TEM and HAADF-STEM results, we created and optimized the corresponding structural model for A-LSM (110) and B-LSM (110), where $\mathrm{La} / \mathrm{Sr}$ atom occupies an A site of A-LSM and Mn atom partly occupies an A-site of B-LSM. Based on the Langmuir-Hinshelwood (L-H) mechanism, ${ }^{[45]}$ namely that $\mathrm{O}_{2}$ and $\mathrm{CO}$ are first adsorbed at the surface of a solid catalyst, and then adsorbed $\mathrm{CO}\left(\mathrm{M}-\mathrm{CO}^{*}\right)$ can react with activated adsorbed oxygen $\left(\mathrm{M}-\mathrm{O}^{*}\right)$ to form the final product $\mathrm{CO}_{2}\left(\mathrm{CO}^{*}+\mathrm{O}^{*} \rightarrow\right.$ $\mathrm{CO}_{2(\mathrm{~g})}$ ), energy profiles and reaction pathway for $\mathrm{CO}$ oxidation at the surface of A-LSM and B-LSM were calculated in order to probe possible structure-activity relationships. It is well known that the activation of an oxygen molecule and of lattice oxygen both play a key role in the $\mathrm{CO}$ oxidation reaction for transition metal oxide catalysts. ${ }^{46-48}$ The activation pathway for the $\mathrm{O}_{2}$ molecule proceeds via the following steps: $\mathrm{O}_{2}(\mathrm{~g}) \rightarrow \mathrm{O}_{2}^{-}$(superoxide species) $\rightarrow \mathrm{O}_{2}{ }^{2-}$ $\left(\mathrm{TS}\right.$, transition state) $\rightarrow \mathrm{O}^{-}\left(\mathrm{M}-\mathrm{O}^{*}\right) .^{[45,49,50]}$ As displayed in Figure 6, in the $\mathrm{O}_{2}$ dissociation pathway, the two steps for $\mathrm{O}_{2} \rightarrow \mathrm{O}_{2}^{-}$and $\mathrm{O}_{2}{ }^{2-} \rightarrow \mathrm{O}^{-}$are exothermic reactions and the relative energy is negative, and they have only a small effect on the catalytic process. However, the transformation from adsorbed $\mathrm{O}_{2}^{-}$(1) to transition state $\mathrm{O}_{2}{ }^{2-}$ (2) needs to overcome an 
activation energy barrier (Figure 6). The perovskite surface usually contains suitable superoxide species and engineering of the surface electronic structure of perovskite catalysts to decrease this energy barrier is a significant research topic. ${ }^{[41]}$ Our calculations reveal that Mn-terminated LSM (B-LSM) possesses lower energy $(\Delta \mathrm{E}=0.8 \mathrm{eV})$ than does conventional $\mathrm{La} / \mathrm{Sr}$-terminated LSM (A-LSM, $\Delta \mathrm{E}=1.54 \mathrm{eV}$ ), and this is consistent with our experimental results for O 1s XPS, O K-edge XAS, $\mathrm{H}_{2}$-TPR, and $\mathrm{O}_{2}$-TPD which demonstrated that surface Mn segregations improve the activity of surface lattice oxygen and optimize the hybrid orbitals between $\mathrm{Mn} 3 \mathrm{~d}$ and $\mathrm{O} 2 \mathrm{p}$ in perovskite LSM. These changes facilitate the transformation from ordinary lattice oxygen $\mathrm{O}^{2-}$ to active lattice oxygen $\mathrm{O}^{2-x}$ and the formation of more unsaturated bonds, which drastically decreases the activation energy of the intermediate $\mathrm{O}_{2}^{2-}$ species. Furthermore, in the process for $(4) \rightarrow(5)$, the $\mathrm{Mn}$ atom occupying an $\mathrm{A}$ site exhibits larger energy $(\Delta \mathrm{E}=-0.07 \mathrm{eV})$ than does an $\mathrm{La} / \mathrm{Sr}$ atom $(\Delta \mathrm{E}=-0.53 \mathrm{eV})$.

However, in the process for $(6) \rightarrow(7)$, A-LSM and B-LSM produced different calculation results for the same reactive sites for B-site Mn sites of LSM so that B-LSM displays lower energy $(-1.14 \mathrm{eV},-1.47 \mathrm{eV})$ than A-LSM $(-0.95 \mathrm{eV},-0.94 \mathrm{eV})$ for the process $\mathrm{M}-\mathrm{O} * \rightarrow \mathrm{M}-\mathrm{CO}_{2}{ }^{*} \rightarrow \mathrm{M}+\mathrm{CO}_{2}$. These results suggest that the active $\mathrm{Mn}$ ions at the A site may not be the important active center and the main role of $\mathrm{MnO}_{x}$ segregation is to tune the hybrid orbital between the Mn 3d and O $2 \mathrm{p}$ in perovskite LSM and improve the activity of the surface oxygen site. In addition, the adsorption energies for the $\mathrm{O}_{2}$ and $\mathrm{CO}$ molecules in the crystal facet (100) of A-LSM and B-LSM were also calculated and the optimized structures are shown in Figures S30-S31. The calculated results showed that the Mn-terminated B-LSM (100) exhibits lower adsorption energies for the $\mathrm{O}_{2}$ and $\mathrm{CO}$ molecules compared with La/Sr-terminated A-LSM (100). Furthermore, in the reaction pathways for $\mathrm{O}_{2}{ }^{*} \rightarrow \mathrm{O}_{2}{ }^{*}$ and $\mathrm{O}_{2}{ }^{*} \rightarrow \mathrm{OO}^{*}, \mathrm{~B}-\mathrm{LSM}(100)$ also displays a lower relative energy compared to A-LSM (100). 
Therefore, the Mn-terminated surface possesses a higher activation ability for oxygen species compared with an $\mathrm{La} / \mathrm{Sr}$ terminated surface.

Based on the above experimental and calculation results, we can conclude that tailored $\mathrm{MnO}_{x}$ segregation in LSM creates more self-regenerating surface-active sites and decreases the activation energy barrier of the oxygen species compared with traditional A-LSM, which improves the activity and stability of perovskite LSM during the $\mathrm{CO}$ oxidation reaction. The root cause of this phenomenon is that the Mn segregation can optimize the hybrid orbital between $\mathrm{Mn} 3 \mathrm{~d}$ and $\mathrm{O} 2 \mathrm{p}$ and improve the activity of the surface oxygen sites $\left(\mathrm{O}^{2-}\right.$ to $\left.\mathrm{O}^{2-x}\right)$.

\section{Conclusion}

In summary, we report a novel strategy for engineering highly active Mn-terminated perovskite manganese oxides by a one-pot hydrothermal method. We demonstrate that surface termination in the B-LSM sample is due to active $\mathrm{MnO}_{x}$ segregation rather than conventional $\mathrm{AO}_{x}$ segregation. Furthermore, we also investigated the contribution of various factors for surface B-site termination in perovskite oxides and found that the hydrothermal environment and the perovskite non-stoichiometry $(\mathrm{A}<\mathrm{B})$ were instrumental, with non-stoichiometry and the high-pressure hydrothermal environment facilitating the growth of Mn atoms at the outer surface at the end of the hydrothermal reactions. Regulating the two synthetic conditions not only creates a significant $\mathrm{MnO}_{x}$ segregation but also significantly reduce the particle size of the perovskite manganate. By using HAADF-STEM, XAS, XPS, and SRPES, we investigated the unusual surface electronic structure of the perovskite B-LSM where the A-site at the outer surface is occupied by a low-valence Mn atom and where Mn-excess is subsequently constructed into active $\mathrm{MnO}_{x}$ phases. This greatly improves the activity of the surface lattice oxygen from inert $\mathrm{O}^{2-}$ to active $\mathrm{O}^{2-x}$ and creates more self-regenerating surface active sites. DFT calculations confirmed that the enhanced activity for B-LSM relates to the decrease of the activation energy barriers from absorbed $\mathrm{O}_{2}^{-}$species to absorbed $\mathrm{O}^{-}$species in the $\mathrm{CO}$ 
oxidation process. Therefore, perovskite B-LSM exhibits Pt-like catalytic activity and outstanding durability for $\mathrm{CO}$ oxidation. This work effectively surmounts the fundamental flaw that an ideal bulk-like terminated surface in perovskite oxides is dominated by ineffectual A-site cations and provides important insights into the key role played by surface lattice oxygen during the catalytic reaction.

\section{Supporting Information}

Supporting Information is available from the Wiley Online Library or from the author.

\section{Acknowledgements}

We thank Professors Lin Gu and Qinghua Zhang for helpful measurements using a spherical aberration correction electron microscope. This work was supported by the National Natural Science Foundation of China (21831003, 21671076, 21621001, and 21801090), the Natural Science foundation of Jilin Province (No.20191004020TC), the Fundamental Research Funds for the Central Universities (3102018bzc007), and the China Postdoctoral Science Foundation (No. 2019M661203). The authors thank Beamline BL14W1 (Shanghai Synchrotron Radiation Facility) and NSRL beamline BL12B-a (National Synchrotron Radiation Laboratory) for providing beam time. We thank the Photoemission Endstations (BL10B) in National Synchrotron Radiation Laboratory (NSRL) for help with characterization. R. Cao acknowledges support from the DOE funded LDRD program and SSRL. The calculations have been performed using the Vienna ab-initio simulation package VASP.6.1.0. CR thanks the EPSRC for an overseas travel grant.

Received: ((will be filled in by the editorial staff))

Revised: ((will be filled in by the editorial staff)) Published online: ((will be filled in by the editorial staff))

\section{References}

[1] X. G. Chen, X. J. Song, Z. X. Zhang, H. Y. Zhang, Q. Pan, J. Yao, Y. M. You, R. G. 
Xiong, J. Am. Chem. Soc. 2020, 142, 6117.

[2] M. Yen, Y. H. Lai, C. Y. Kuo, C. T. Chen, C. F. Chang, Y. H. Chu, Adv. Funct. Mater. 2020, 2004597.

[3] S. Royer, D. Duprez, F. Can, X. Courtois, C. Batiot-Dupeyrat, S. Laassiri, H. Alamdari, Chem. Rev. 2014, 114, 10292.

[4] B. Keimer, S. A. Kivelson, M. R. Norman, S. Uchida, J. Zaanen, Nature 2015, 518, 179.

[5] C. H. Kim, G. Qi, K. Dahlberg, W. Li, Science 2010, 327, 1624.

[6] J. Zhu, H. Li, L. Zhong, P. Xiao, X. Xu, X. Yang, Z. Zhao, J. Li, ACS Catal. 2014, 4, 2917.

[7] D. Neagu, T. S. Oh, D. N. Miller, H. Menard, S. M. Bukhari, S. R. Gamble, R. J. Gorte, J. M. Vohs, J. T. Irvine, Nat. Commun. 2015, 6, 8120.

[8] W. Si, Y. Wang, Y. Peng, J. Li, Angew. Chem. 2015, 127, 8065.

[9] L. Cao, W. Liu, Q. Luo, R. Yin, B. Wang, J. Weissenrieder, M. Soldemo, H. Yan, Y. Lin, Z. Sun, Nature 2019, 565, 631.

[10] Y. Zhu, W. Zhou, J. Yu, Y. Chen, M. Liu, Z. Shao, Chem. Mater. 2016, 28, 1691.

[11] Y. Zhu, L. Zhang, B. Zhao, H. Chen, X. Liu, R. Zhao, X. Wang, J. Liu, Y. Chen, M. Liu, Adv. Funct. Mater. 2019, 29, 1970236.

[12] B.-J. Kim, X. Cheng, D. F. Abbott, E. Fabbri, F. Bozza, T. Graule, I. E. Castelli, L. Wiles, N. Danilovic, K. E. Ayers, N. Marzari, T. J. Schmidt, Adv. Funct. Mater. 2018, 28, 1804355 .

[13] S. Gupta, W. Kellogg, H. Xu, X. Liu, J. Cho, G. Wu, Chem. Asian J. 2016, 11, 10.

[14] L. Yang, G. Yu, X. Ai, W. Yan, H. Duan, W. Chen, X. Li, T. Wang, C. Zhang, X. Huang, J.-S. Chen, X. Zou, Nat. Commun. 2018, 9, 5236.

[15] J. Druce, H. Tellez, M. Burriel, M. D. Sharp, L. J. Fawcett, S. N. Cook, D. S. McPhail, T. Ishihara, H. H. Brongersma, J. A. Kilner, Energy Environ. Sci. 2014, 7, 3593.

[16] Y. Li, W. Zhang, Y. Zheng, J. Chen, B. Yu, Y. Chen, M. Liu, Chem. Soc. Rev. 2017, 46, 6345.

[17] H. G. Lee, L. Wang, L. Si, X. He, D. G. Porter, J. R. Kim, E. K. Ko, J. Kim, S. M. Park, B. Kim, A. T. S. Wee, A. Bombardi, Z. Zhong, T. W. Noh, Adv. Mater. 2020, 32, 1905815. [18] D. Neagu, G. Tsekouras, D. N. Miller, H. Menard, J. T. Irvine, Nat. Chem. 2013, 5, 916. [19] A. K. Opitz, A. Nenning, C. Rameshan, R. Rameshan, R. Blume, M. Hävecker, A. Knop-Gericke, G. Rupprechter, J. Fleig, B. Klötzer, Angew. Chem. Int. Edit. 2015, 54, 2628.

[20] Z. Du, H. Zhao, S. Yi, Q. Xia, Y. Gong, Y. Zhang, X. Cheng, Y. Li, L. Gu, K. Swierczek, ACS Nano 2016, 10, 8660.

[21] Y. Liu, H. Dai, J. Deng, L. Zhang, Z. Zhao, X. Li, Y. Wang, S. Xie, H. Yang, G. Guo, Inorg. Chem. 2013, 52, 8665.

[22] Y. F. Sun, Y. Q. Zhang, J. Chen, J. H. Li, Y. T. Zhu, Y. M. Zeng, B. S. Amirkhiz, J. Li, B. Hua, J. L. Luo, Nano Lett. 2016, 16, 5303.

[23] G. M. Rupp, A. K. Opitz, A. Nenning, A. Limbeck, J. Fleig, Nat. Mater. 2017, 16, 640.

[24] N. Tsvetkov, Q. Lu, L. Sun, E. J. Crumlin, B. Yildiz, Nat. Mater. 2016, 15, 1010.

[25] W. Jung, H. L. Tuller, Energy Environ. Sci. 2012, 5, 5370.

[26] F. Polo-Garzon, S. Z. Yang, V. Fung, G. S. Foo, E. E. Bickel, M. F. Chisholm, D. E. Jiang, Z. Wu, Angew. Chem. 2017, 56, 9820.

[27] J. M. Martirez, S. Kim, E. H. Morales, B. T. Diroll, M. Cargnello, T. R. Gordon, C. B. 
Murray, D. A. Bonnell, A. M. Rappe, J. Am. Chem. Soc. 2015, 137, 2939.

[28] A. Ohtomo, H. Y. Hwang, Nature 2004, 427, 423.

[29] S. V. Ovsyannikov, A. M. Abakumov, A. A. Tsirlin, W. Schnelle, R. Egoavil, J. Verbeeck, G. Van Tendeloo, K. V. Glazyrin, M. Hanfland, L. Dubrovinsky, Angew. Chem. 2013, 52, 1494.

[30] M.-R. Li, P. W. Stephens, M. Croft, Z. Deng, W. Li, C. Jin, M. Retuerto, J. P. Hodges, C. E. Frank, M. Wu, D. Walker, M. Greenblatt, Chem. Mater. 2018, 30, 4508.

[31] A. Prodi, E. Gilioli, A. Gauzzi, F. Licci, M. Marezio, F. Bolzoni, Q. Huang, A. Santoro, J. W. Lynn, Nat. Mater. 2004, 3, 48.

[32] D. R. Modeshia, R. I. Walton, Chem. Soc. Rev. 2010, 39, 4303.

[33] K. Huang, W. Feng, L. Yuan, J. Zhang, X. Chu, C. Hou, X. Wu, S. Feng, CrystEngComm 2014, 16, 9842.

[34] R. D. Johnson, D. D. Khalyavin, P. Manuel, L. Zhang, K. Yamaura, A. A. Belik, Phys. Rev. B 2018, 98, 104423.

[35] F. Tao, M. E. Grass, Y. Zhang, D. R. Butcher, J. R. Renzas, Z. Liu, J. Y. Chung, B. S. Mun, M. Salmeron, G. A. J. S. Somorjai, Science 2008, 322, 932.

[36] D. Gao, Y. Zhang, Z. Zhou, F. Cai, X. Zhao, W. Huang, Y. Li, J. Zhu, P. Liu, F. Yang, G. Wang, X. Bao, J. Am. Chem. Soc. 2017, 139, 5652.

[37] M. Huynh, C. Shi, S. J. Billinge, D. G. Nocera, J. Am. Chem. Soc. 2015, 137, 14887.

[38] V. R. Galakhov, M. Demeter, S. Bartkowski, M. Neumann, N. A. Ovechkina, E. Z. Kurmaev, N. I. Lobachevskaya, Y. M. Mukovskii, J. Mitchell, D. L. Ederer, Phys. Rev. B 2002, 65113102.

[39] X. Wang, K. Huang, J. Qian, Y. Cong, C. Ge, S. Feng, Sci. Bull. 2017, 62, 658.

[40] E. Mutoro, E. J. Crumlin, H. Pöpke, B. Luerssen, M. Amati, M. K. Abyaneh, M. D. Biegalski, H. M. Christen, L. Gregoratti, J. Janek, Y. Shao-Horn, J. Phys. Chem. Lett. 2012, 3,40 .

[41] X. Wang, Z. Pan, X. Chu, K. Huang, Y. Cong, R. Cao, R. Sarangi, L. Li, G. Li, S. Feng, Angew. Chem. 2019, 58, 11720.

[42] H. Yu; X. Wei, J. Li, S. Gu, S. Zhang, L. Wang, J. Ma, L. Li, Q. Gao, R. Si, F. Sun, Y. Wang, F. Song, H. Xu, X. Yu, Y. Zou, J. Wang, Z. Jiang, Y Huang, Nucl. Sci. Tech. 2015, 26, 050102 .

[43] J. Jones, H. Xiong, A. Delariva, E. Peterson, H. Pham, S. Challa, G. Qi, S. Oh, M. Wiebenga, X. Pereira Hernandez, Science 2016, 353, 150.

[44] Z. Zhang, Y. Zhu, H. Asakura, B. Zhang, J. Zhang, M. Zhou, Y. Han, T. Tanaka, A. Wang, T. Zhang, N. Yan, Nat. Commun. 2017, 8, 16100.

[45] J. Yang, S. Hu, Y. Fang, S. Hoang, L. Li, W. Yang, Z. Liang, J. Wu, J. Hu, W. Xiao, C. Pan, Z. Luo, J. Ding, L. Zhang, Y. Guo, ACS Catal. 2019, 9, 9751.

[46] J. Luo, M. Meng, J. Yao, X. Li, Y. Zha, X. Wang, T. Zhang, Appl. Catal., B 2009, 87, 92.

[47] J. van Bokhoven, C. Louis, J. Miller, M. Tromp, O. Safonova, P. Glatzel, Angew. Chem. Int. Ed. 2006, 45, 4651.

[48] N. Weiher, A. Beesley, N. Tsapatsaris, L. Delannoy, C. Louis, J. van Bokhoven, S. Schroeder, J. Am. Chem. Soc. 2007, 129, 2240.

[49] Y. A. Mastrikov, R. Merkle, E. Heifets, E. A. Kotomin, J. Maier, J. Phys. Chem. C 2010, 
$114,3017$.

[50] L. Nie, D. Mei, H. Xiong, B. Peng, Z. Ren, X. I. P. Hernandez, A. DeLaRiva, M. Wang, M. H. Engelhard, L. Kovarik, A. K. Datye, Y. Wang, Science 2017, 358, 1419.

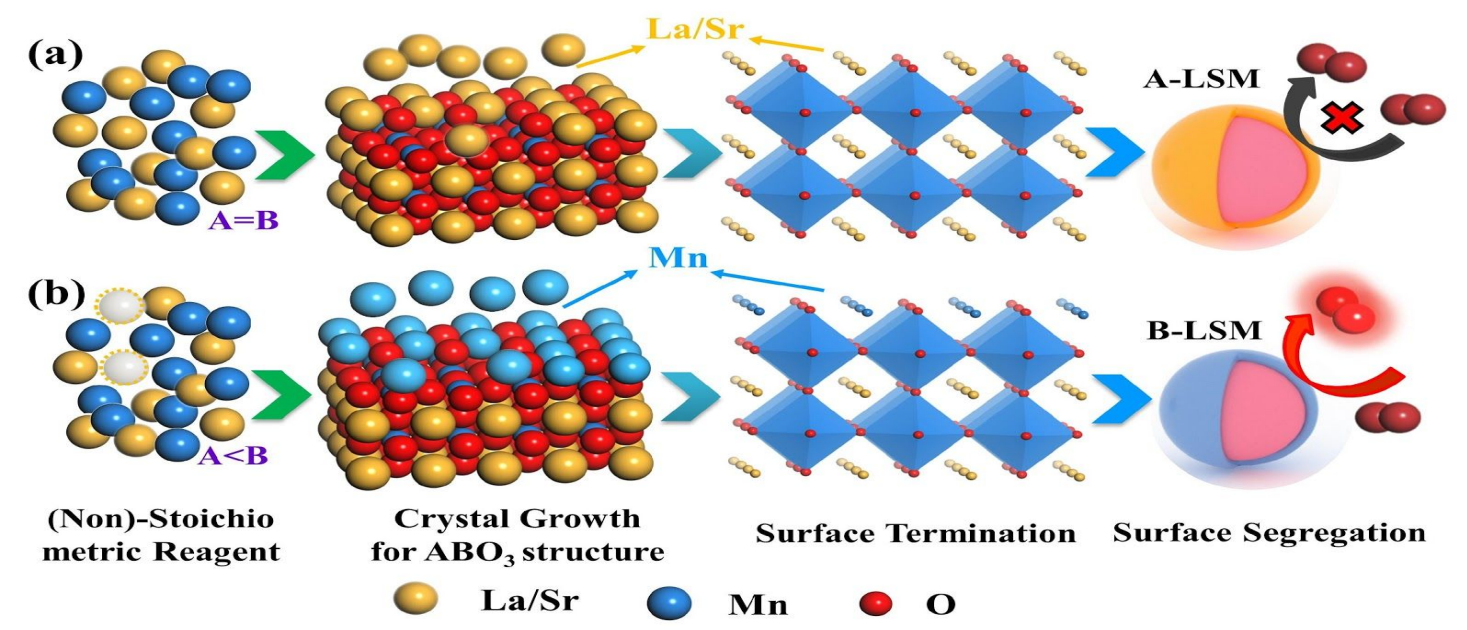

Figure 1. Illustration of the surface termination evolution and surface structure for (a) La/Sr-terminated perovskite A-LSM and (b) Mn-terminated perovskite B-LSM. Blue octahedron: $\mathrm{MnO}_{6}$ octahedron; Pink spheres: bulk perovskite oxides.
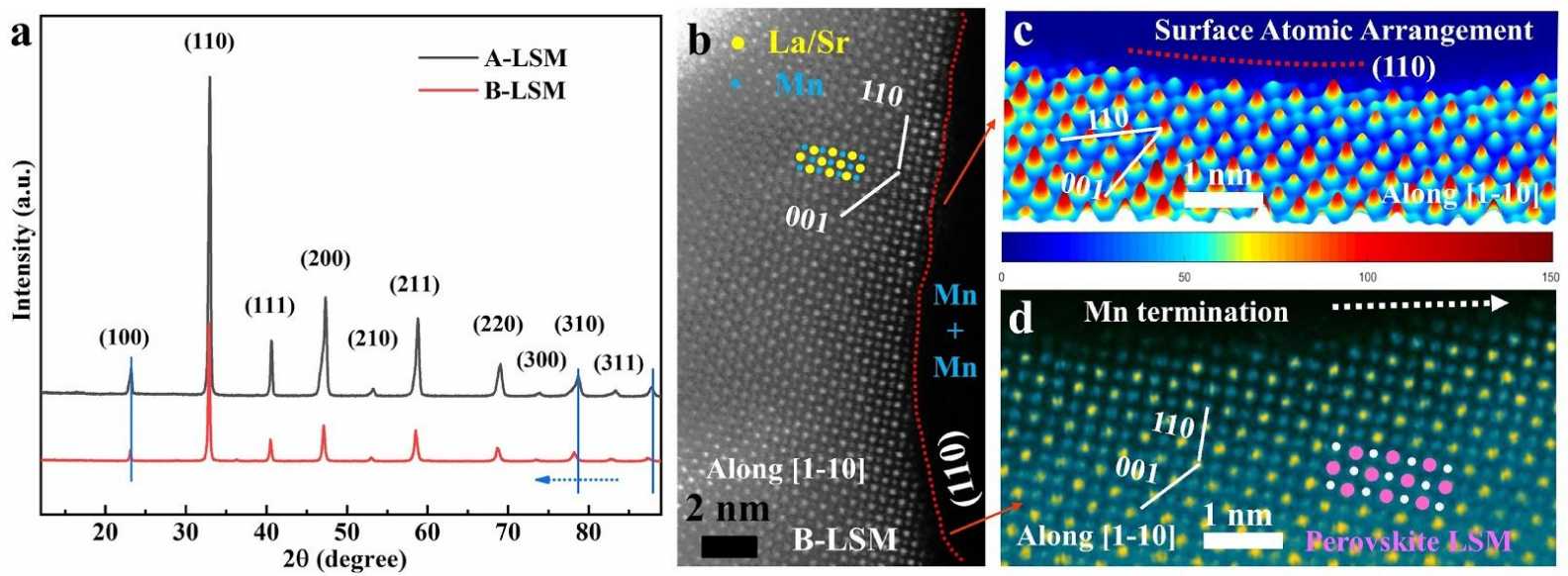

Figure 2. a) X-ray diffraction patterns (XRD) for A-LSM and B-LSM; b) $[1-10]_{\mathrm{p}}$ HAADF-STEM image of B-LSM; c) The corresponding 3D intensity-profile images derived 
from the magnified HAADF-STEM image in (b) by Gaussian fitting for atomic column intensity line profiles of the $\mathrm{La}, \mathrm{Sr}$ and $\mathrm{Mn}$ atoms; and d) Atomic-resolution enlarged HAADF-STEM images on the outer surface of B-LSM viewed along the [1-10] zone axis for clear observation of surface termination, where bright yellow represents heavy atoms $\mathrm{La} / \mathrm{Sr}$ and blue represents $\mathrm{Mn}$ atoms with small ion radius.
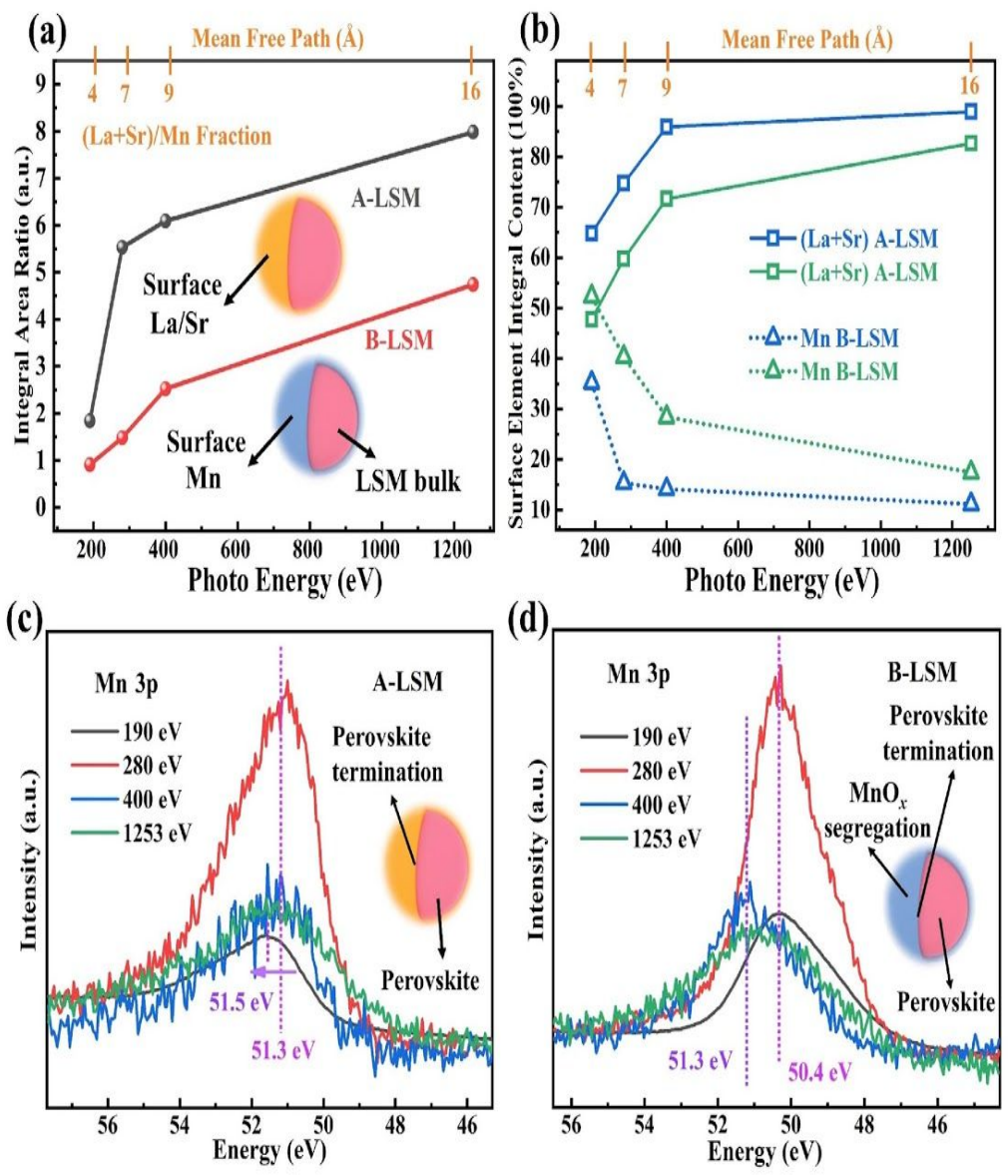

Figure 3. Surface composition and structure. a) Dependence of $(\mathrm{La}+\mathrm{Sr}) / \mathrm{Mn}$ atomic fractions of A-LSM and B-LSM as a function of photoelectron energy and mean free path of the excited photoelectrons; b) Dependence of $\mathrm{La} / \mathrm{Sr}$ and $\mathrm{Mn}$ atomic fractions of A-LSM and B-LSM as a function of photoelectron energy and mean free path; Mn 3p XPS spectra at different photo energy for c) A-LSM and d) B-LSM. Schematics show the surface compositions and termination of A-LSM and B-LSM, but do not represent their shape. Detected photoelectrons with a photo energy of $\sim 190 \mathrm{eV}$ derived from the outer surface of perovskite LSM. The signal of photoelectrons with $\sim 280, \sim 400$, and $\sim 1253 \mathrm{eV}$ can be contributed to surface atomic layers at different depths. The y-axis data points have an associated error of (a) \pm 0.2 and (b) \pm 3 . 

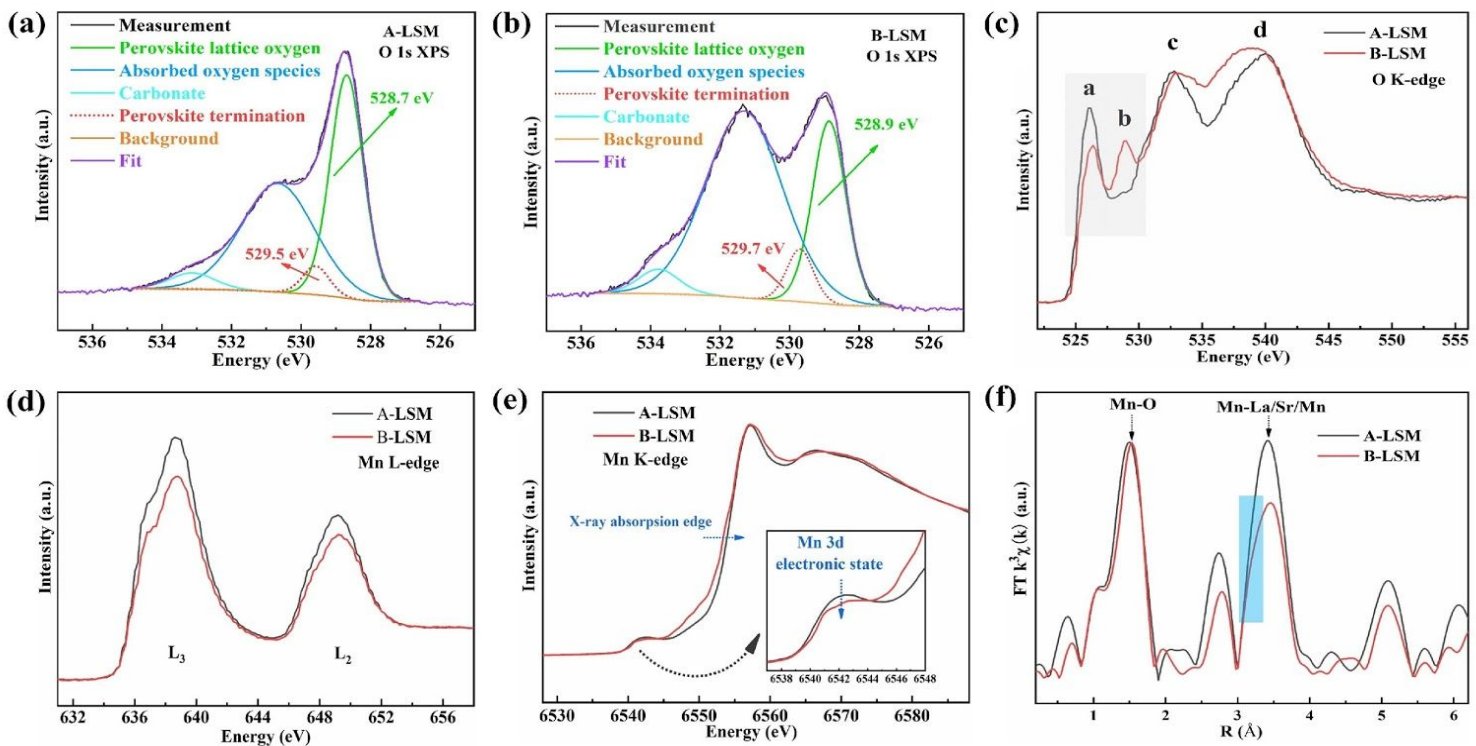

Figure 4. Electronic structure and valence bond information. a) $\mathrm{O}$ K-edge soft X-ray absorption spectra, b) Mn L-edge soft X-ray absorption spectra, c) Mn K-edge X-ray absorption near edge structure (XANES), and d) R-space Fourier-transformed FT $\left(\mathrm{k}^{3} \chi(\mathrm{k})\right)$ of Mn K-edge EXAFS for A-LSM and B-LSM.
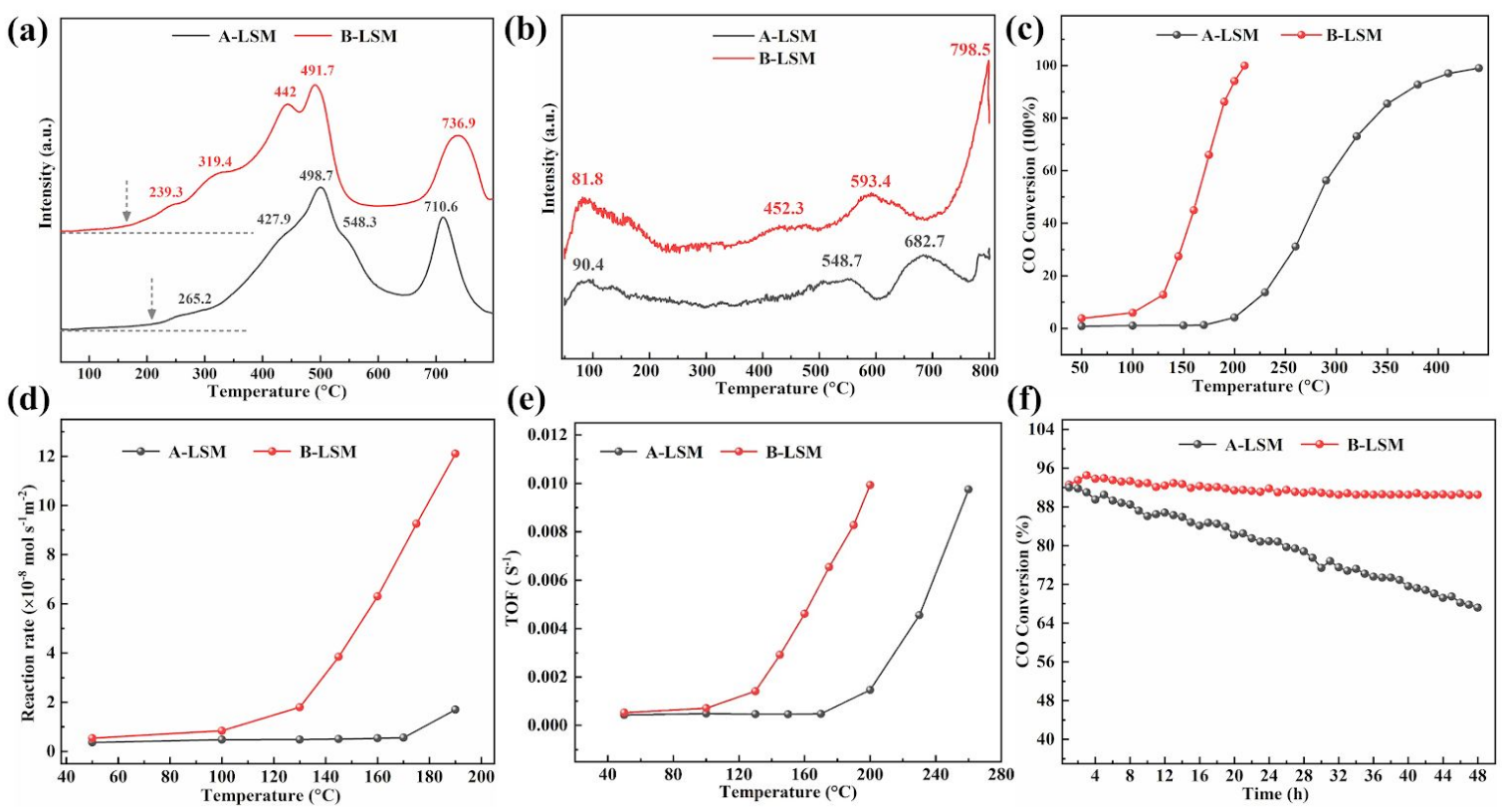

Figure 5. Catalytic performance characterization. a) $\mathrm{H}_{2}-\mathrm{TPR}$ profiles, b) $\mathrm{O}_{2}-\mathrm{TPD}$ profiles, c) CO oxidation activity, d) Reaction rate normalized by catalyst surface area, e) Temperature-dependent turnover frequencies (TOFs) of CO oxidation normalized by the 
number of Mn active sites at the catalyst surface, f) Stability test for CO oxidation activity at $\sim 90 \%$ conversion for $48 \mathrm{~h}$ for A-LSM and B-LSM.

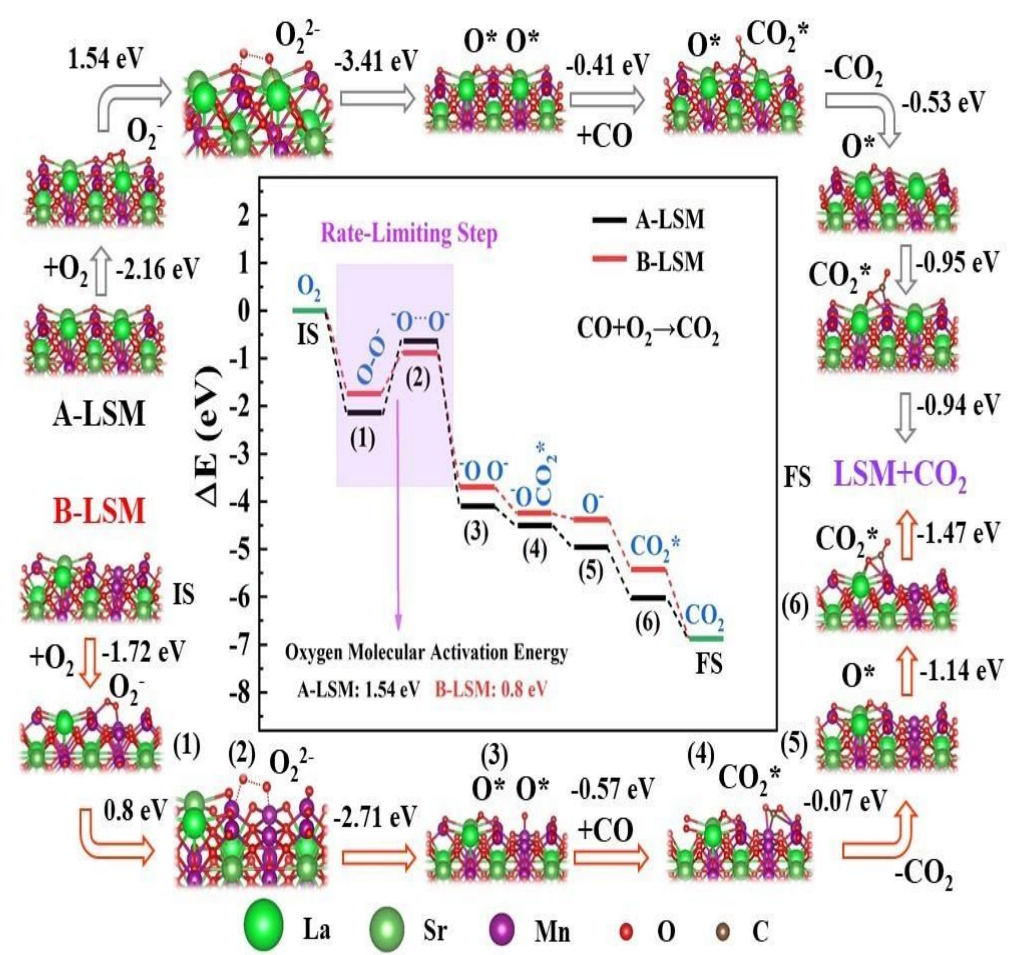

Figure 6. Calculated energy profiles and reaction pathway for $\mathrm{CO}$ oxidation at the surface of A-LSM and B-LSM via a Langmuir-Hinshelwood mechanism.

Table 1. XPS, ICP, EDS, BET Surface Areas, $\mathrm{H}_{2}$ Consumption, and CO Oxidation Activity for A-LSM and B-LSM

\begin{tabular}{|c|c|c|c|c|c|c|c|c|c|c|}
\hline \multirow[t]{2}{*}{ sample } & \multicolumn{2}{|c|}{ XPS } & & & & & & \multicolumn{3}{|c|}{$\begin{array}{c}\text { Catalytic } \\
\text { activity }\end{array}$} \\
\hline & $\begin{array}{c}(\mathrm{La}+\mathrm{Sr}) \\
/ \mathrm{Mn}\end{array}$ & $\begin{array}{r}\text { Oads/ } \\
\text { Olatt } \\
r\end{array}$ & $\begin{array}{l}(\mathrm{La}+\mathrm{Sr}) \\
\quad / \mathrm{Mn} \\
\text { r ratio }\end{array}$ & $\begin{array}{c}\mathrm{Sr} / \\
(\mathrm{La}+\mathrm{Sr})\end{array}$ & $\begin{array}{c}(\mathrm{La}+\mathrm{Sr}) \\
/ \mathrm{Mn}\end{array}$ & $\begin{array}{l}\text { surface } \\
\text { area } \\
{\left[\mathrm{m}^{2} \mathrm{~g}^{-1}\right]}\end{array}$ & $\begin{array}{c}\mathrm{H}_{2} \\
\text { consumption } \\
{\left[\mathrm{mmol} \mathrm{g}^{-1}\right]}\end{array}$ & $\mathrm{T}_{10}$ & $\begin{array}{c}\mathrm{T}_{50} \\
{\left[{ }^{\circ} \mathrm{C}\right]}\end{array}$ & $\mathrm{T}_{90}$ \\
\hline A-LSM & 1.76 & 1.26 & 1.08 & 0.49 & 1.46 & 0.85 & 2.02 & 218 & 283 & 368 \\
\hline B-LSM & 0.95 & 2.73 & 0.93 & 0.52 & 1.24 & 2.65 & 2.39 & 117 & 164 & 196 \\
\hline
\end{tabular}


To address the problem of inert surface $\mathrm{AO}_{x}$ segregation in perovskite oxides, high-efficiency B-site terminated perovskite manganate has been prepared via one-pot hydrothermal method, and this improves both activity and durability. This $\mathrm{MnO}_{x}$ termination resulting from low-valent $\mathrm{Mn}$ ions occupying an $\mathrm{A}$ site at the outer surface facilitates the activation of surface oxygen sites, and dramatically decreases the activation energy barriers associated with oxygen species.

Xiyang Wang, Xinbo Li, Xuefeng Chu, Rui Cao, Jingyu Qian, Yingge Cong, Keke Huang, Jiaao Wang, Carl Redshaw, Ritimukta Sarangi, Guangshe Li, and Shouhua Feng*

Manipulating Surface Termination of Perovskite Manganate for Oxygen Activation

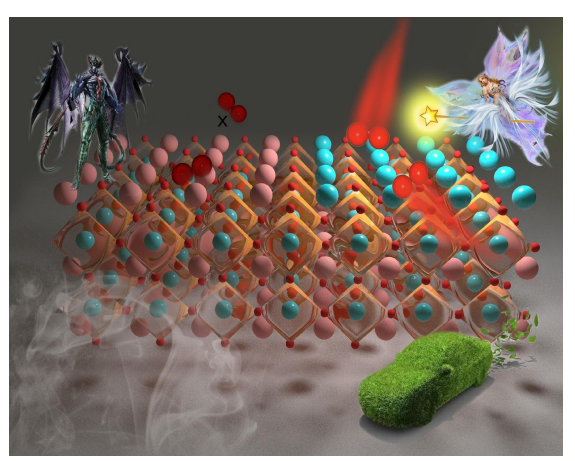

